ISBN 978-81-933894-1-6

5th International Conference on Chemical, Agricultural, Biological and Environmental Sciences

(CAFES-17)

Kyoto (Japan) April 18-19, 2017

\title{
Design and Performance Analysis of Novel Shear Enhanced Module for The Recovery of PVA from Desizing Waste Water
}

\author{
Mithu Naskar ${ }^{1}$, Kalpana Biswas ${ }^{1}$, Chiranjib Bhattacharjee ${ }^{2}$, Debasish Sarkar ${ }^{1 *}$ \\ ${ }^{1}$ Department of Chemical Engineering, University of Calcutta, Kolkata: 700009, India \\ ${ }^{2}$ Department of Chemical Engineering, Jadavpur University, Kolkata; 700032, India
}

\begin{abstract}
Polyvinyl alcohol (PVA) is an important sizing agent in textile industries. As textile effluent containing PVA is considered to be hazardous water pollutant, so several attempts has been taken to recover the same. Amongst all separation processes, Shear enhanced membrane separation have been adopted as one of the standard techniques to be used to recover PVA from desizing waste water generated by textile industries. The present work is an attempt to analyze the performance of an indigenously designed novel shear enhanced module, named as Intermeshed Spinning Basket Module (ISBM) with inbuilt hydrodynamic cleaning facility. The analysis has been undertaken with different basket rotational speed $(\Omega)$ and transmembrane pressure (TMP) in ultrafiltration of simulated desizing wastewater using polyvinylidene fluoride (PVDF) membranes of $100 \mathrm{kDa}$ molecular weight cut-off (MWCO). Permeate flux as high as $1.46 \times 10-5 \mathrm{~m} 3 \mathrm{~m}-2 \mathrm{~s}-1$ was observed for a TMP of $4.41 \mathrm{~atm}$ and at rotational speed of $200 \mathrm{rpm}$. The regenerated flux after the first cleaning exhibits $28-58 \%$ enhancement compared to the corresponding steady state flux at the end of first normal run. The result shows the efficacy of the module for the recovery of PVA
\end{abstract}

Keywords- Intermeshed spinning basket module, Permeate flux, Regression analysis, Shear enhanced module, cleaning run.

\section{Introduction}

Indian textile industry contributes about $4 \%$ to gross domestic product (GDP) and $17 \%$ to the country's export earnings, according to the Annual Report 2010-11 published by the Ministry of Textiles (Gera 2012). Different type of chemicals and large amount of water is utilized by these textile industries for dry/wet processing. Earlier to the weaving stage, sizing agents, such as polyvinyl alcohol (PVA) (Ozdemir et al. 2011) is added for smoothening the fibers and to enhance the thread tenacity (Lee et al. 1999). After the weaving stage, sizing agents must be removed from cloths when it undergoes finishing operation. In this stage, chemicals are removed by washing the clothes with hot water, which produces an effluent stream, commonly known as desizing waste water. Effluent generated by desizing operation contribute largely to the biochemical oxygen demand (BOD), approximately $45 \%$ of total BOD load 
(Porter et al. 1972). For treating the desizing waste water, amongst all the processes membrane based separation techniques have been adopted as an efficient effluent treatment processes. However, the processes suffer from a severe drawback of massive flux decline with time due to the reversible accumulation of rejected solute particles known as concentration polarization which turns into further severe when the solute particle permanently clogs the pore known as fouling. Fouling permanently reduces the hydrodynamic permeability of the membrane and simultaneously affects the lifetime of a membrane. So to alleviate the problem several modifications may be noted that because of these two non idealities, permeate flux decreases from startup to the respective steady state value. Regeneration of the fouled membrane can only be done either by back flushing/ back washing or by chemical cleaning (Kazemimoghadam and Mohammadi 2007). Another type of method shear enhanced module was also named as spinning basket membrane (SBM) module (Sarkar et al. 2012; Sarkar et al. 2012; Sarkar et al. 2012; Sarkar et al. 2012) having self cleaning techniques, giving uninterrupted normal run, avoiding the usage of chemicals and also rather, increasing the total lifetime of membranes and consequently, cut down the economics of the process. But the proposed SBM module (consist of four radial arms) cannot give the total release from concentration polarization due to lower surface area. The present work has been undertaken in an attempt to indigenously develop and characterize a novel DSE module, named as Intermeshed Spinning Basket Module (ISBM), considering its inherent similarity with the well know spinning basket reactor. However, the present module consists of two spinning basket, intermeshed with each other. The module has been characterized under different basket rotational speed $(\Omega)$ and transmembrane pressure (TMP) in ultrafiltration of simulated desizing wastewater $\left(\mathrm{PVA} / \mathrm{water}, \mathrm{C}_{0}=1.14\right.$ $\mathrm{kg} \mathrm{m}^{-3}$ ), using polyvinylidene fluoride (PVDF) membranes of $100 \mathrm{kDa}$ molecular weight cut-off (MWCO).

\section{Experimental}

\subsection{Materials and analysis}

A simulated desizing wastewater [Polyvinyl alcohol (cold, average molecular weight: $125000 \mathrm{~g} \mathrm{~mol}$

${ }^{1}$ )/water solution] was used as feed. The PVA (cold) was obtained from E. Merck, Mumbai, India. Moist semi-permeable, asymmetric PVDF membranes of pore size (MWCO: $100 \mathrm{kDa}$ ) were obtained from Koch Membrane Systems (USA). The rectangular flat sheet membrane was operable in the $\mathrm{pH}$ range of 2.5-10.5. Typical operating pressure for the membrane was 210-830 $\mathrm{kPa}$ (maximum operating pressure: $970 \mathrm{kPa}$ ) with operating temperature range of $5-54^{\circ} \mathrm{C}$ (Maximum operating temperature: $65.5^{\circ} \mathrm{C}$ ). The concentration of polyvinyl alcohol (PVA) in the feed, retentate and permeate, was measured with a hand held refractometer (Model PAL-85S, Atago Co. Ltd., Japan). The density and viscosity of PVA solution were measured by using pycnometer and Ostwald viscometer, respectively.

\subsection{Membrane System and Operation}

The present module has been named as Intermeshed Spinning Basket Membrane (ISBM) module solely designed by us, was fabricated by EMCEE, Howrah, West Bengal (India). It consists of a couple of two identical spinning baskets mounted on two parallel hollow shafts driven by induction motor, fitted with a variable speed drive (ABB IP20/UL open type) with suitable belt-pulley, which can also revert the direction of rotation (Fig. 1). Each basket consists of four radial arms, fitted with flat sheet membranes (effective filtration area: $105 \times 55 \mathrm{~mm}^{2}$ ) on one side, while the other side is impermeable. The baskets are intermeshed through two identical spur gears, mounted on parallel shafts. The phase difference of rotation between two baskets is fixed at $45^{\circ}$. The whole system with suitable sealing arrangements is placed in an oval shaped stainless steel tank and fed by a triplex piston pump. 

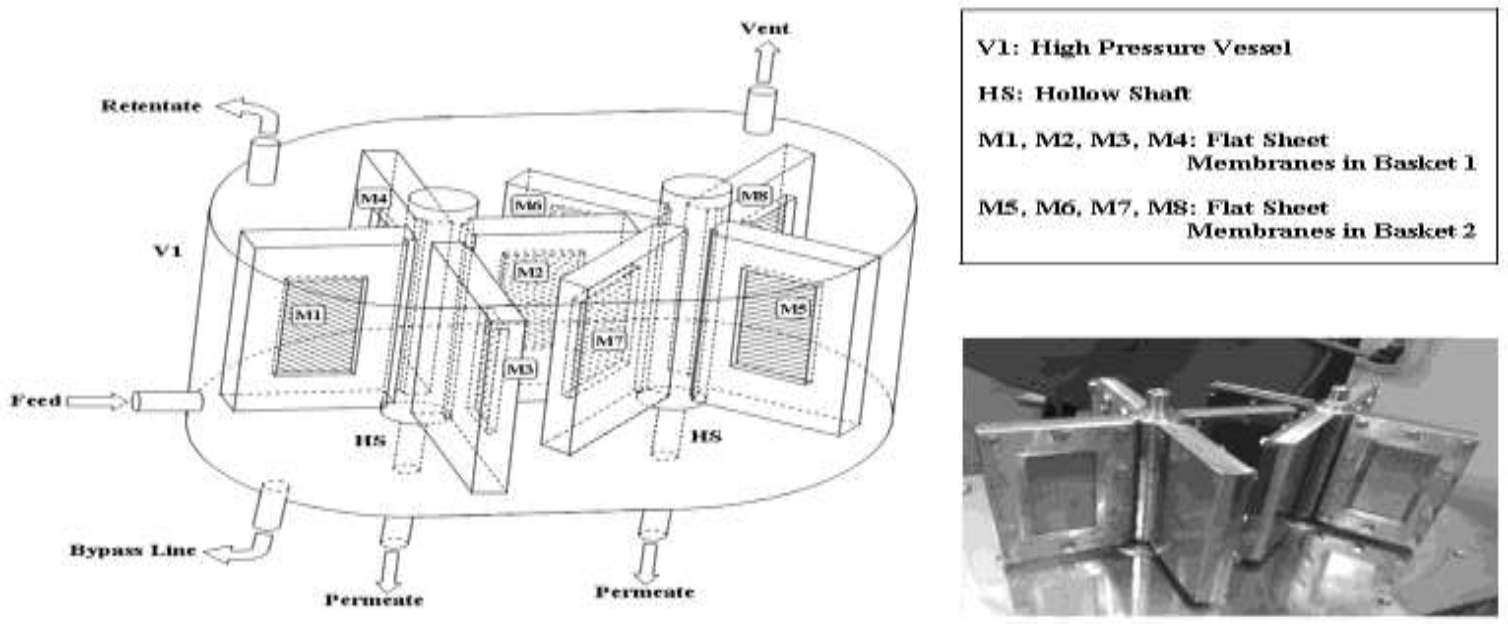

Fig.1 Schematic of proposed ISBM module

The baskets are subjected to rotate at equal speed and in the opposite direction, towards there spective membrane area vectors. This leads to a high membrane shear rate and consequently a high flux. The proposed module was operated at cleaning mode when the flux decline was observed. For the cleaning operation, back pressure regulator has to be fully opened in order to release the TMP and baskets are rotated in the reverse direction. Due to reverse directional rotation, a local vacuum is expected to set in over the membrane surface. Accordingly, a back pressure (from permeate to retentate side) will come into play, which will displace the accumulated solute on the membrane surface, which reduces the hydrodynamic resistance of the membrane and thereby recover the permeate flux in the next normal run.

\subsection{Experimental Procedure}

Initially, the membrane was compacted with distilled water at a transmembrane pressure (higher than the highest operational pressure) of $6 \mathrm{~kg} . \mathrm{cm}^{-2}$ for $4 \mathrm{~h}$ until the water flux attained a steady state value. The performance study was done with respect to three different process parameters, which are (1) applied TMP (2) rotational speed of the basket $(\Omega)$ and (3) feed concentration $\left(\mathrm{C}_{0}\right)$. Retentate flow rate was held constant (8 LPM) and feed concentration was fixed at $1.14 \mathrm{~kg} \mathrm{~m}^{-3}$ as the reported concentration of PVA in desizing wastewater is about the same for all the experimental runs. Once the permeate flux attains a steady value and remains unchanged over a prolong interval, the module was switched to cleaning.

Each normal run was conducted for $9 \mathrm{~h}$. On the other hand, every cleaning run was conducted arbitrarily for $300 \mathrm{~s}$, as the run time duration is impossible to optimize a priori. Every experimental run consists of three successive normal runs, intervened by two cleaning runs. The same procedure was repeated for each experiment with a fixed set TMP, $\Omega$ and $\mathrm{C}_{0}$ values. The experiments were carried out varying the parameters in the following range: TMP $\left(\mathrm{kg} . \mathrm{cm}^{-2}\right) \Rightarrow[2.5,4], \Omega(\mathrm{rpm}) \Rightarrow[100,350]$ and $\mathrm{C}_{0}$ $(\mathrm{v} / \mathrm{v} \%) \Rightarrow[3,6]$.

\section{Results and Discussion:}

\subsection{Variation of permeate flux with time under different transmembrane pressure and rotational speed}

Fig. 2 represents the variation of transient permeate flux, $J(t)$ for the first two normal runs, intervened by a single cleaning run for different TMP and $\Omega$. As stated earlier, fig. 3 clearly indicates the drop of permeate flux with time for both the normal runs. For example, at TMP $=4.41 \mathrm{~atm}$ and $\Omega=200 \mathrm{rpm}$, the steady permeate flux, at the end of the first normal run was $36 \%$ reduced relative to its initial counterpart. The same was only $10.7 \%$ in case of the second normal run. However, the result does not mean the 
second normal run to be more efficient one than the first. Actually, the regenerated permeate flux after the first cleaning run was $81 \%$ of the corresponding initial flux with the same parametric conditions.

In general, the permeate flux was observed to increase with TMP as well as $\Omega$. For a change of TMP from 1.59 to $4.41 \mathrm{~atm}$, the steady permeate flux changes by $89 \%$ at $200 \mathrm{rpm}$, which alternatively indicates the respective gradient $\left[=\frac{\partial \boldsymbol{J}}{\partial(\text { TMP })}\right]$ to be $1.46 \times 10^{-5} \mathrm{~m}^{3} \mathrm{~m}^{-2} \mathrm{~s}^{-1} \mathrm{~atm}^{-1}$ at $200 \mathrm{rpm}$. On the other hand, the steady flux changes by $7.26 \%$ for a change of $\Omega$ from 100 to $200 \mathrm{rpm}$ under fixed condition of TMP $(=4.41 \mathrm{~atm})$. Therefore the respective gradient $\left[=\frac{\partial \mathrm{J}}{\partial \Omega}\right]$, at TMP $=4.41 \mathrm{~atm}$ becomes $1.43 \times 10^{-8} \mathrm{~m}^{3} \mathrm{~m}^{-2} \mathrm{~s}^{-1}$ $\mathrm{rpm}^{-1}$. Regarding the cleaning run, fig. 3 indicates that over the indicated range of TMP and $\Omega$, as used in the present study, 28-58\% regeneration (with respect to the flux values at the end of the first normal run) was achieved in the first cleaning run. In the second cleaning run, 33-60\% enhancement was noticed. It may also be noted that on an average, the regenerated permeate flux, even after the second cleaning run was close to $60 \%$ of the corresponding initial flux values at the time of start up.

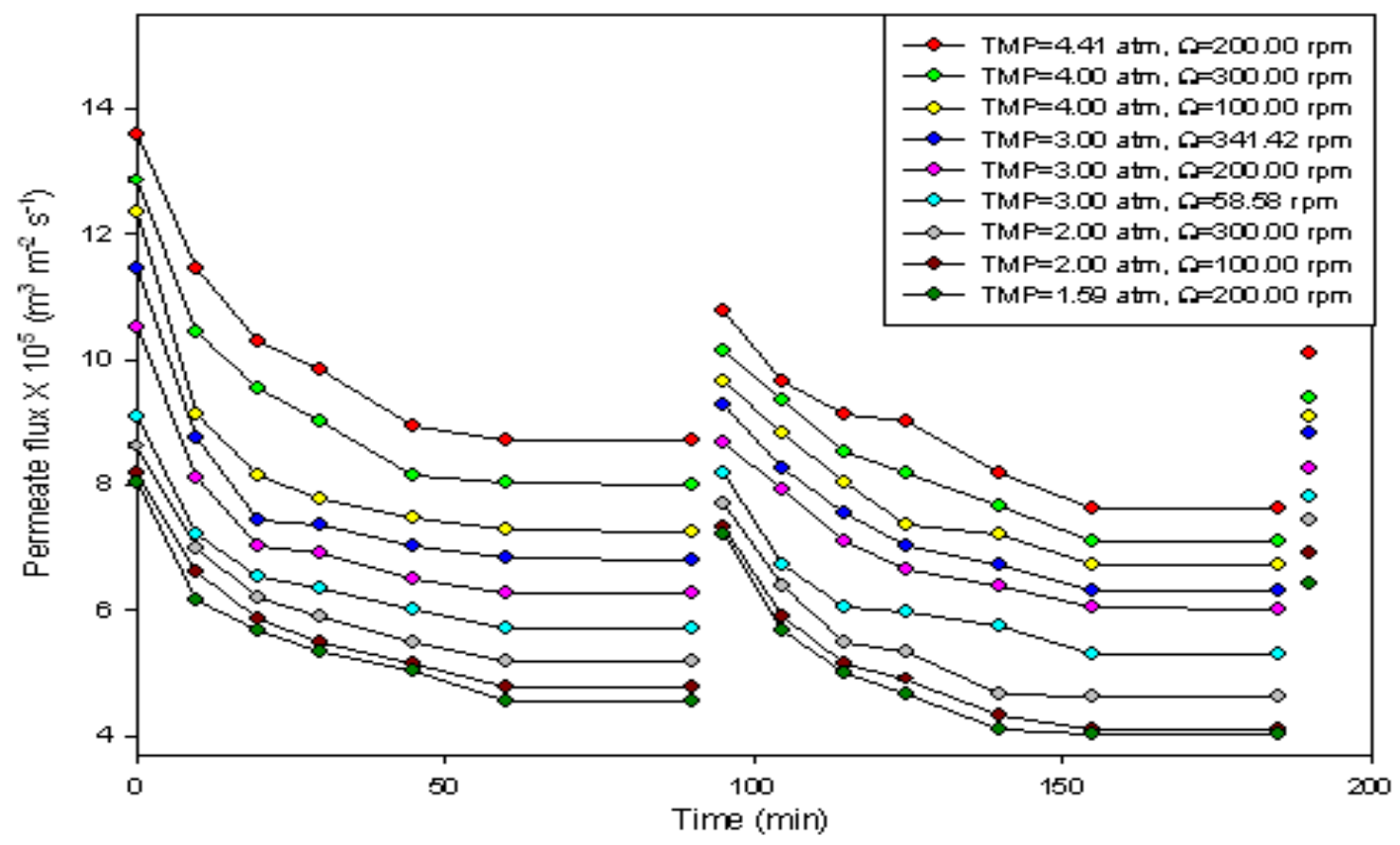

Fig. 2 Transient variation of permeate flux at different TMP and $\Omega$

In this respect, it's worth noting that the steady flux, at the end of the first normal run was only $50 \%$ of the same initial flux. The result clearly points out the novelty of the present design, as the regenerated flux value after $190 \mathrm{~min}$ is even higher than the normal flux at the end of $90 \mathrm{~min}$ only (without cleaning run).

\section{Conclusion}

As in the present module, two spinning baskets are intermeshed together; it has been named as Intermeshed Spinning Basket Membrane (ISBM) module. The results indicate that the present ISBM module is able to regenerate the permeate flux of $28-58 \%$ of its start up value after 9 hours of continuous operation. The maximum permeate flux was observed to be as high as $1.46 \times 10^{-5} \mathrm{~m}^{3} \cdot \mathrm{m}^{-2} \cdot \mathrm{s}^{-1}$ in separation of PVA from desizing using a PVDF membrane. Therefore, the result shows the efficacy of the newly designed module in the recovery of PVA from desizing waste water.

\section{Acknowledgements}


The Financial assistance, provided by Department of Science and Technology (DST), Govt. of India, under Women Scientist Scheme (Ref. No.: SR/WOS-A/ET-89/2016 dated 17/11/2016) is gratefully acknowledged.

\section{Reference}

[1] N. Gera, "Significance and Future Prospects of Textile Exports in Indian Economy,". International Research Journal, vol-2, pp.1-16 2012.

[2] C. Ozdemir, M.K. Oden, , S. Sahinkaya, and E. Kalipci, "Color Removal from Synthetic Textile Wastewater by Sono-Fenton Process," Clean-Soil Air Water, vol-39 (1), pp.60-67 2011.

[3] S.W. Lee, S.J. Haam, J.H. Jang, and Y.C. Lee, "Ultrafiltration of Desizing Wastewater Containing PVA in Bench Scale Test,” Environmental Technology, vol-20, pp.277-283 1999.

[4] J.J. Porter, D.W. Lyons, and W.F. "Nolan Water Uses and Wastes in the Textile Industry," Environmental Science Technology, vol-6 (1), pp.36-41 1972.

[5] M. Kazemimoghadam, and T. Mohammadi, "Chemical cleaning of ultrafiltration membranes in the milk industry," Desalination, vol-204, pp.213-218 2007.

[6] A. Sarkar, D. Sarkar, and C. Bhattacharjee, "Design and Performance Characterization of a new shear enhanced module with inbuilt cleaning arrangement," Society of Chemical Industry, vol-87, pp.1121-1130, 2012.

[7] A. Sarkar, D. Sarkar, M. Gupta and C. Bhattacharjee, "Recovery of Polyvinyl Alcohol from Desizing Wastewater Using a Novel High-Shear Ultrafiltration Module," Clean - Soil Air Water, Vol. 40, pp. 830-837, August 2012. https://doi.org/10.1002/clen.201100527

[8] D. Sarkar, A. Sarkar, A. Roy and C. Bhattacharjee, "Performance characterization and design evaluation of spinning basket membrane (SBM) module using computational fluid dynamics (CFD)," Separation and Purification Technology, Vol. 94, pp. 23-33, June 2012. https://doi.org/10.1016/j.seppur.2012.03.034

[9] . Sarkar, S. Moulik, D. Sarkar, A. Roy and C. Bhattacharjee, "Performance characterization and CFD analysis of a novel shear enhanced membrane module in ultrafiltration of Bovine Serum Albumin (BSA)," Desalination, Vol. 292, pp. 53-63, April 2012.

https://doi.org/10.1016/j.desal.2012.02.009 